\title{
Store-Load-Branch (SLB) Predictor: A Compiler Assisted Branch Prediction for Data Dependent Branches
}

\author{
M. Umar Farooq Khubaib Lizy K. John \\ Department of Electrical and Computer Engineering \\ The University of Texas at Austin \\ ufarooq@utexas.edu,khubaib@ece.utexas.edu,ljohn@ece.utexas.edu
}

\begin{abstract}
Data-dependent branches constitute single biggest source of remaining branch mispredictions. Typically, data-dependent branches are associated with program data structures, and follow store-load-branch execution sequence. A set of memory locations is written at an earlier point in a program. Later, these locations are read, and used for evaluating branch condition. Branch outcome depends on data values stored in data structure, which, typically do not have repeatable pattern. Therefore, in addition to history-based dynamic predictor, we need a different kind of predictor for handling such branches.

This paper presents Store-Load-Branch (SLB) predictor; a compiler-assisted dynamic branch prediction scheme for data-dependent direct and indirect branches. For every data-dependent branch, compiler identifies store instructions that modify the data structure associated with the branch. Marked store instructions are dynamically tracked, and stored values are used for computing branch flags ahead of time. Branch flags are buffered, and later used for making predictions. On average, compared to standalone TAGE predictor, combined TAGE+SLB predictor reduces branch MPKI by $21 \%$ and $51 \%$ for SPECINT and EEMBC benchmark suites respectively.
\end{abstract}

\section{Introduction}

Most branch prediction techniques rely on branch history information for predicting future branches. Some use short history [11] [21] [36], while others use longer history [16] [25] [30] [32]. History-based dynamic branch prediction schemes have shown to reach high prediction accuracy for all except few hard-to-predict branches. Figures 1 and 2 show branch mispredictions per $1 \mathrm{~K}$ instructions for EEMBC and SPECint benchmark suites using several history-based branch predictors. As can be seen from the figures, several benchmarks have higher branch mispredictions even when using long history branch predictor.
This work is based on the following observation: Hardto-predict data-dependent branches are commonly associated with program data structures such as arrays, linked lists, trees etc., and follow store-load-branch execution sequence similar to one shown in listing 1. A set of memory locations is written while building and updating the data structure (line 2, listing 1). During data structure traversal, these locations are read, and used for evaluating branch condition (line 7, listing 1).

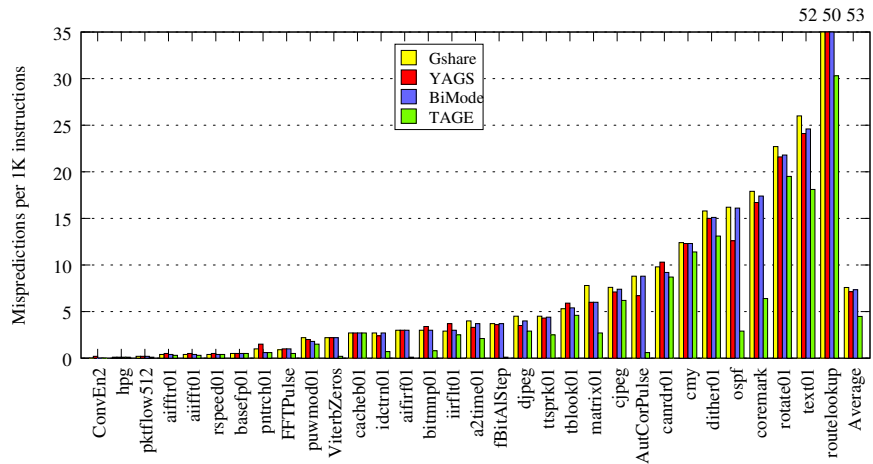

Figure 1. MPKI for EEMBC benchmark suite

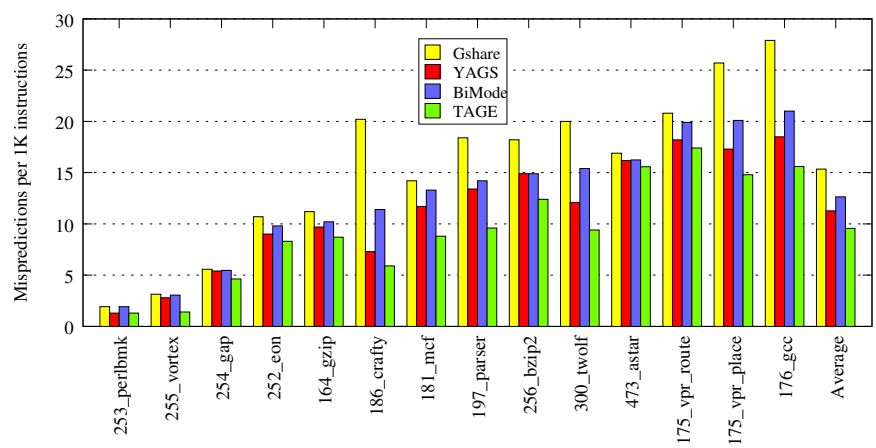

Figure 2. MPKI for SPECint benchmark suite

This paper proposes Store-Load-Branch (SLB) predictor, a compiler-assisted dynamic branch prediction scheme 
Listing 1. Store-Load-Branch execution sequence

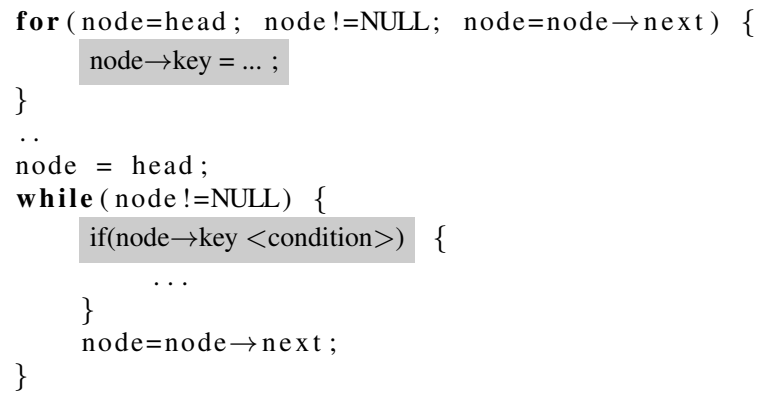

for data-dependent branches using data value correlation. Compiler identifies all program points where data structure associated with a hard-to-predict data-dependent branch is referenced and modified. At run-time, hardware tracks marked store instructions that modify the data structure, computes branch condition flags ahead of time using store data values, and buffers them in a structure at store addresses. Later, during data structure traversal, pre-computed flags are read using predicted load address, and used for predicting branch outcome.

Typically, instruction that loads data structure values is quickly followed by branch instruction for evaluating branch condition using loaded values. Therefore, actual load address is usually not available before branch instruction gets fetched. Hence, we use predicted load address for reading pre-computed branch flags. Addresses for simple data structures such as arrays are easier to predict using stride-based address predictor. Bekerman et al. proposed load address predictor for irregular data structures such as linked list and tree [4], which we adapted according to our requirement.

We compared our design with the state-of-the-art TAGE branch predictor [32]. Results show that, for several benchmarks, top mispredicting branches in the TAGE predictor are accurately predicted using SLB predictor. On average, compared to standalone TAGE predictor, combined TAGE+SLB predictor reduces branch mispredictions per $1 \mathrm{~K}$ instructions (MPKI) by 21\% for SPECint [34] benchmark suite. Similarly, for EEMBC [12] benchmark suite, MPKI is reduced by $51 \%$.

This paper makes following contributions:

1. We investigate program patterns that manifest hard-topredict, data-dependent branches.

2. We propose SLB prediction scheme for datadependent branches, which predicts branch outcome using pre-computed branch flags.

3. Our implementation of SLB requires adding couple of hint instructions to ISA, and light-weight hardware structures.
Rest of the paper is organized as follows: Section 2 presents motivating examples from real benchmarks. Section 3 describes SLB prediction scheme. Simulation methodology and results are presented in Sections 4 and 5 respectively. Section 6 discusses related work. Finally, Section 7 concludes the paper.

Listing 2. LD-BR execution sequence (cjpeg)

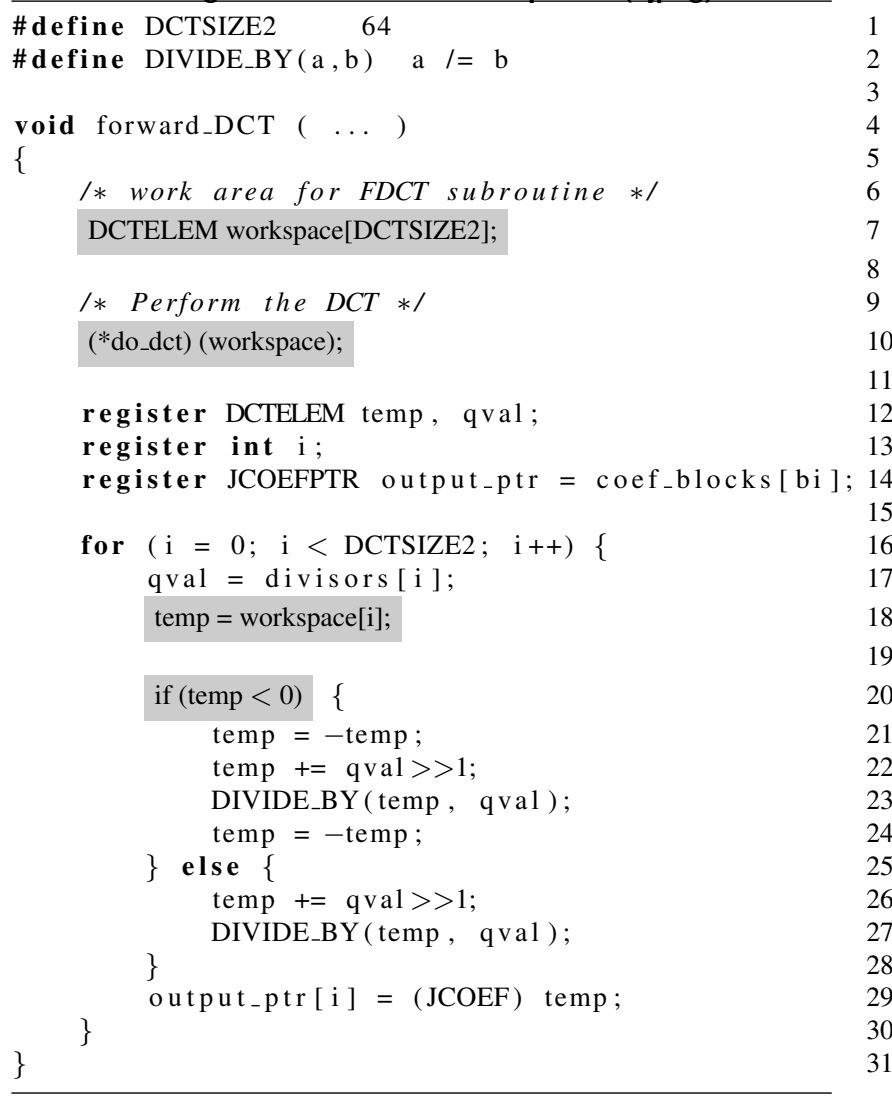

\section{Motivating Examples}

\subsection{Example 1: Data-Dependent Direct Branches}

We will use cjpeg benchmark from eembc-consumer suite as a motivating example. The benchmark performs standard JPEG compression on a given image. Input image is broken into block of $8 \times 8$ pixels, and each block goes through discrete cosine transform (DCT), quantization and entropy coding steps. As shown in listing 2, do_dct function (line 10) populates an array, workspace, with DCT coefficients whose values range between -1024 to 1023 . During quantization, each DCT coefficient is read (line 18), compared (line 20) to see if it is positive or negative, and quantized accordingly. The branch 'if (temp $<\mathbf{0})$ ' (line 20) is 
Listing 3. ST execution sequence (cjpeg)

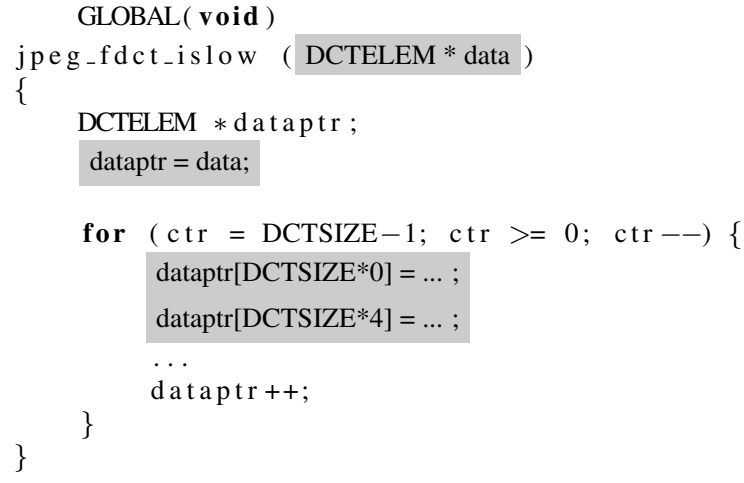

an example of a hard-to-predict data-dependent branch. Table 1 (row 3) shows branch characteristics and prediction accuracy of this branch using short and long history predictors. It shows that using longer history TAGE predictor does not improve prediction accuracy for this branch. Instead, if branch condition flags are computed and buffered while populating array workspace in do_dct function $^{1}$ (see listing 3, lines 8-9), a simple buffer lookup can yield perfect branch prediction.

\subsection{Example 2: Data-Dependent Indirect Branches}

Indirect branches are generally harder to predict than direct branches as they may have multiple targets corresponding to a single static indirect branch.

Listing 4 shows a ray tracer program, Eon, taken from SPECint2000 suite. Each ray must be tested for intersection with all the objects in the scene. A scene consists of several different types of objects with a common base class mrSurface, as shown in the listing 4 (lines 1-8). While reading the scene, these objects are stored into a 3D datastructure called grid using its mrGrid::insert method (line 10). The grid is later traversed in the mrGrid::viewingHit method (line 21), to see if the incoming ray hits any object in the grid. During each iteration of the while loop, the next object's pointer (oPtr) is read from the grid (line 36), and depending on the type of the object, corresponding viewingHit() method is invoked (line 41). Since the sequence of objects stored in the grid does not have a repeatable pattern, predicting target address for virtual function call (line 41) using history-based indirect branch predictor results in lower prediction accuracy (see table 1, row 4). Instead, if viewingHit() function addresses corresponding to different types of objects in the grid are buffered while inserting ob-

\footnotetext{
${ }^{1}$ do_det is a pointer function, and is assigned as: $\mathrm{fdct} \rightarrow \mathrm{do}$ ded $=$ jpeg_fdct_islow;
}

jects in the grid (line 15 and 18), a buffer lookup during grid traversal yields correct function address corresponding to the read object. Note that, in addition to accurately predicting target address for 'oPtr $\rightarrow$ viewingHit()' function call (line 41), another hard-to-predict branch at line 37, 'if ( oPtr )', can also be accurately predicted since it also depends on the same object read from the grid.

Listing 4. ST-LD-BR execution sequence (eon)

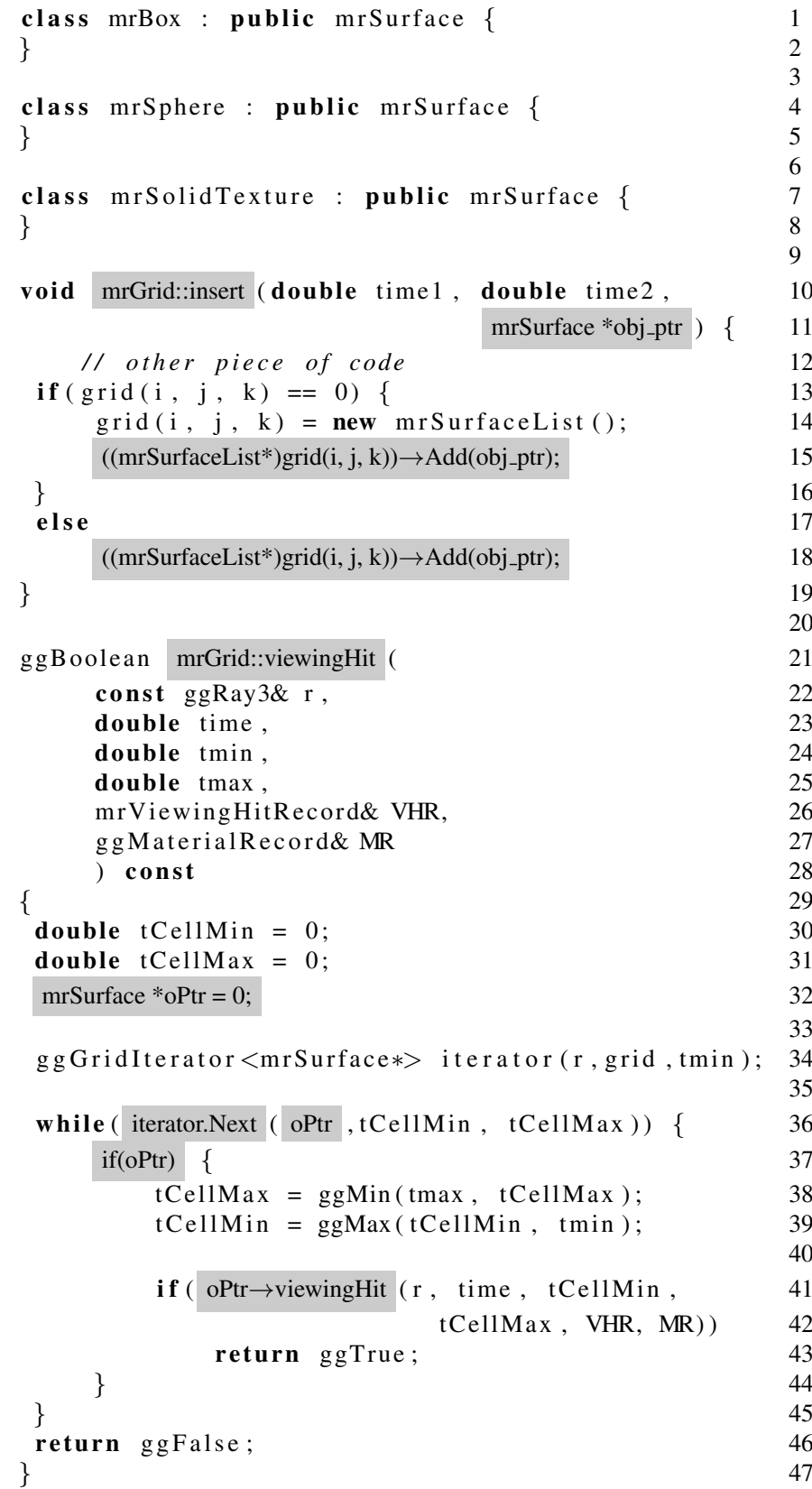




\begin{tabular}{|c|c|c|c|c|c|c|c|}
\hline Benchmark & Br. location & Dynamic count & Taken count & Mispredict count & \multicolumn{2}{|c|}{ Miss rate } \\
\hline & & & & Bi-Mode & TAGE & Bi-Mode & TAGE \\
\hline cjpeg & line 20 & 576000 & $297216(51.6 \%)$ & 282816 & 274889 & $49.1 \%$ & $47.7 \%$ \\
\hline 252.eon & line 41 & 573306 & $573306(100 \%)$ & 251636 & 247841 & $43.8 \%$ & $43.2 \%$ \\
\hline 252.eon & line 37 & 884626 & $311320(35.2 \%)$ & 258697 & 120297 & $29.2 \%$ & $13.5 \%$ \\
\hline
\end{tabular}

Table 1. Characteristics and prediction accuracy of hard-to-predict branches in motivating examples

\section{Store-Load-Branch (SLB) Predictor}

\subsection{Overview}

SLB predictor is a compiler-assisted dynamic branch prediction technique specifically targeted at improving prediction accuracy of data-dependent branches. Most datadependent branches are associated with program data structures such as array, linked list, tree etc. During traversal, these branches operate on elements of data structure. Branch outcome depends on data values stored in the structure, which, typically do not have repeatable patterns. Therefore, instead of relying on branch history information, we compute branch flags and use them for predicting branch direction. Due to deep processor pipeline, data values (and resulting branch flags) are often not available before branch instruction gets fetch. Therefore, instead of using load values, we compute branch flags ahead of time using store values while updating the data structure.

\subsection{Implementation Details}

\subsubsection{Compiler and Architecture Support}

For a data-dependent branch, SLB scheme relies on compiler to identify its store-load-branch (ST-LD-BR) sequence. Starting with branch instruction, compiler identifies load instruction(s) on which branch is dependent. It then identifies store instruction(s) feeding load instruction(s). ST-LD-BR sequence for a branch is encoded and passed down to hardware using special load_hint (HLD) and store_hint (HST) instructions shown in figure 3.

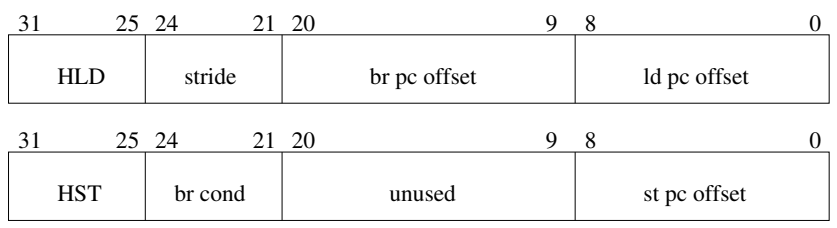

Figure 3. load_hint and store_hint instruction format

Compiler inserts an HLD/HST instruction for every static load/store associated with the branch. Lower 9 bits encode load/store pc offset from HLD/HST instruction. HLD bits 20:9 specify branch pc offset from HLD instruction. On seeing an HLD/HST instruction, hardware uses pc offset values for computing absolute address for load or store instruction associated with the branch instruction. HLD bits 24:21 specify load stride value. HST bits 24:21 specify condition code for evaluating branch outcome at store time.

\subsubsection{Hardware Support}

For identifying ST-LD-BR sequence at run time, hardware provides three main tables, the store table, the load table, and the branch table, as shown in figure 4.

Populating Load Table: On executing an HLD instruction, an entry is created in load table if it does not already exist. 'Tag' field in load table is populated with lower 12 bits of load instruction address which is computed using 'ld pc offset' in HLD instruction. ' $\mathrm{Br}$ pc' field is populated with 12 bits of branch instruction address which is computed using 'br pc offset' in HLD instruction. Rest of the fields in load table are associated with load address predictor, and are explained later.

Populating Store Table: Similar to load table, on executing an HST instruction, an entry is created in store table. 'Tag' field is populated with lower 12 bits of store instruction address which is computed using 'st pc offset' in HST instruction. ' $\mathrm{Br}$ cond' field is populated with 4-bit branch condition code specified in HST instruction.

Populating Branch Table: 'Tag' field in branch table is populated with lower 12 bits of branch instruction address which is computed using 'br pc offset' in HLD instruction.

Once ST-LD-BR sequence is populated in the tables, computing and consuming branch flags can begin.

Computing Branch Flags at Store Time: During code generation, compiler inserts compare instruction prior to store instruction for comparing store value with branch condition. This compare instruction sets the flag register. When store instruction executes and matches store table tag, branch flag is computed using flag register value and corresponding branch condition code in store table. Computed branch flag is stored in T/NT prediction table at store address. For more information on condition codes, flag register and compare/branch instruction, see ARMv7-A architectural manual [2].

Consuming Branch Flags at Fetch Time: During data structure traversal, when a data-dependent branch matches 


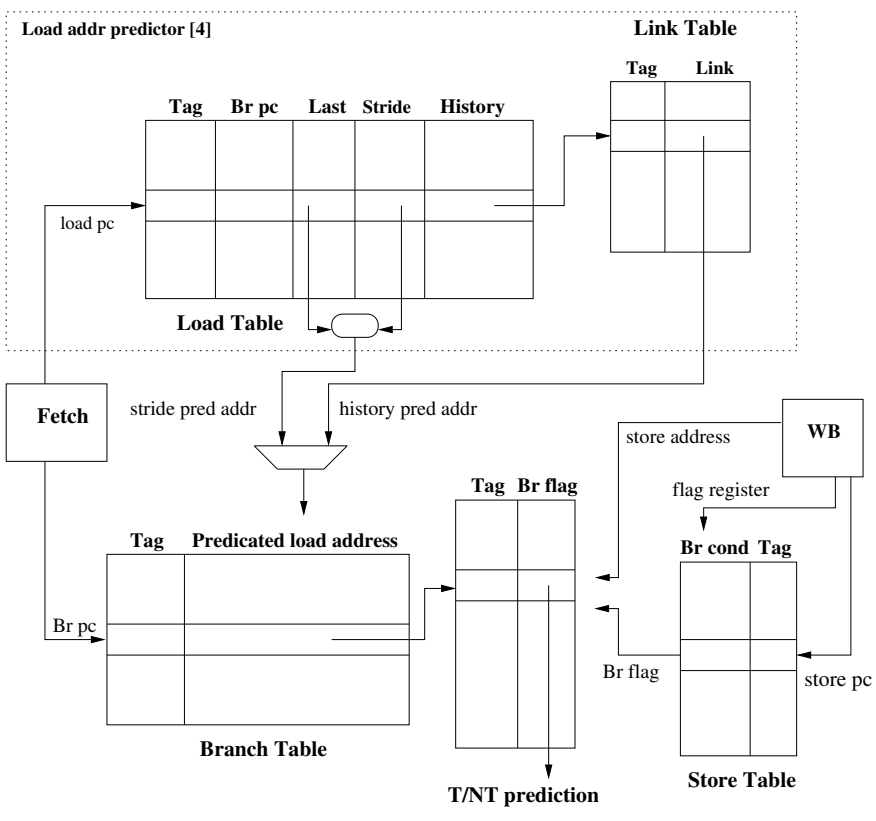

Figure 4. SLB predictor block diagram

branch table tag, prediction table is looked up using predicted load address for making taken/not-taken prediction. Predicted load address is generated using load instruction which appears earlier in program order than branch instruction, therefore, load address prediction is not on critical path of making branch prediction.

Generating Predicted Load Address: Since branch prediction is made early in the pipeline, and data structure traversal often occurs in tight loop, 'predicted load address' is used for accessing T/NT prediction table. Figure 4 (dotted box) shows load address predictor, adapted from Bekerman's load address predictor [4]. Address prediction was originally proposed for reducing load instruction latency. In this paper, we propose an alternate use of load address predictors: using predicted load addresses for predicting data-dependent branches. For regular data structures (e.g. arrays) that are traversed linearly, stride-based load address predictor is sufficient [3][8]. Bekerman et al. proposed advance load address predictor for recursive data structures (e.g. linked lists and trees) [4]. It uses a two-level scheme for predicting the next load address. First level is a perstatic-load table, the load table, where each entry records history of recent addresses seen by the associated load. The history is then used to index a second level table, the link table, which provides the predicted address. Typically, recursive data structure addresses can be accurately predicated by keeping last two addresses in the history. See [4] for more details on load address predictor.

\subsubsection{Support for Indirect Branches}

As oppose to a direct branch, an indirect branch can have multiple targets. Therefore, predicting an indirect branch requires predicting branch target address as oppose to branch direction. Most indirect branch prediction schemes maintain a history of recent targets taken by an indirect branch, and uses history to index into a 'target cache' for predicting the next target address [6] [9] [10] [17]. Similar to direct branches, data-dependent indirect branches typically do not follow history.

SLB indirect branch prediction scheme uses traditional BTB to store multiple targets of an indirect branch at different BTB indices. Figure 5 shows the block diagram for SLB indirect branch target address prediction (only necessary changes from figure 4 are shown).

Populating Store Data Array: When a store instruction executes and matches store table tag, lower 12 bits of store data value is buffered in store data array at store address.

Predicting Branch Target Address: When an indirect branch is fetch, it reads stored data value from store data array using 'predicted load address', hash it with branch pc, and index into branch target buffer (BTB) for predicting branch target address. Different store data values correspond to different targets of an indirect branch, which are stored and subsequently accessed at different BTB indices.

Updating the BTB: If an indirect branch mispredicts, either, because target address is seen for the first time, or it is replaced by another branch target, BTB is updated. Same index computed at BTB lookup time, is used for updating the BTB.

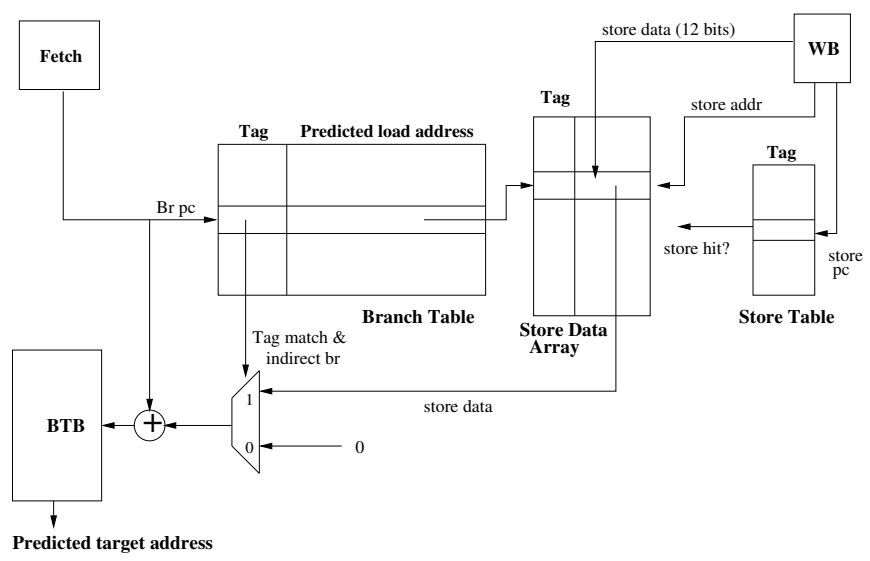

Figure 5. Support for indirect branches

\subsubsection{Choosing Between TAGE and SLB Predictor}

In a combined TAGE+SLB predictor configuration, not every branch is predicted using SLB predictor. If a branch address matches branch table tag in figure 4 , prediction is 


\begin{tabular}{|c|c|c|}
\hline \multicolumn{3}{|c|}{ Load Address Predictor } \\
\hline Load Table & 32 entries x (12+32+32+10+64) & 4800 bits \\
\hline Link Table & $1 \mathrm{k}$ entries x (12+32) & $45056 \mathrm{bits}$ \\
\hline \multicolumn{3}{|c|}{ SLB Conditional Branch Predictor } \\
\hline Branch Table & 32 entries x (12+32) & 1408 bits \\
\hline Store Table & 48 entries x (12+4) & 768 bits \\
\hline T/NT Prediction & $1 \mathrm{k}$ entries x (12+1) & 13312 bits \\
\hline \multicolumn{3}{|c|}{ SLB Indirect Branch Predictor } \\
\hline St. Data Array & 1k entries x (12+12) & 24576 bits \\
\hline
\end{tabular}

Table 2. Implementation cost of SLB predictor

taken from SLB predictor, else prediction is taken from default TAGE predictor.

\subsection{Implementation Cost}

Load, store, and branch tables shown in figure 4 are all per-static-instruction tables, while link table and T/NT prediction table holds dynamic values. We have sized various structures in SLB predictor based on number of SLB branches observed in benchmarks. Table 4 shows number of SLB branches identified in each benchmark along with their associated load and store instructions. Table 2 shows storage cost for implementing SLB predictor ${ }^{2}$. For reducing implementation cost, link table, T/NT table, and store data array in figures 4 and 5 can be made tagless. Total storage required for implementing SLB conditional branch predictor is $63.8 \mathrm{Kbits}$ (39.8 Kbits, if tagless). An additional 24 Kbits (12 Kbits, if tagless) storage is needed for implementing SLB indirect branch predictor.

\section{Experimental Framework}

\subsection{Simulation Methodology}

Results presented in this paper are collected from an ARM performance simulator running benchmarks from EEMBC, SPECint2000 suites [12][34]. In addition, top mispredicting benchmark from SPECint2006 suite, 473.astar, is also included in the experiment. Benchmarks are compiled with ARM RealView compilation tool (RVCT 4.1) [28] with -O3 optimization flag. Our detailed cycle accurate simulator models a superscalar out-of-order processor core with 4-wide, 15 stages integer pipeline, 32KB L1 I and $\mathrm{D}$ cache, and $1 \mathrm{MB} \mathrm{L} 2$ cache. Table 3 shows simulation parameters for the front-end pipeline. We used Bi-Mode branch predictor [21] as our baseline. We then compared the baseline with 1) standalone TAGE predictor [32], and 2) combined TAGE+SLB predictor. We obtain TAGE predictor code from [31] and integrated with our timing simulator.

\footnotetext{
${ }^{2}$ We assume 32 bits for data and address, and 12 bits for tag.
}

Table 3. Front-end pipeline parameters

\begin{tabular}{|l|l|}
\hline Instruction Fetch & 4-wide, 5-deep; \\
\hline BTB & 2048-entry; \\
\hline Return Address Pred & 16-entry; \\
\hline \multicolumn{2}{|c|}{ Conditional Branch Predictors } \\
\hline Bi-Mode (baseline) & $\begin{array}{l}\text { 48 Kbits, taken/not-taken/sel each 8k-entry; } \\
\text { 13-bit global history register; }\end{array}$ \\
\hline TAGE & 64 Kbits, 14 components; 130 bit max hist; \\
\hline SLB & 68 Kbits, 32-entry load table; \\
& 32-entry branch table; 48-entry store table; \\
& 1K-entry T/NT table; 1K-entry link table; \\
\hline \multicolumn{2}{|c|}{ Indirect Branch Predictors } \\
\hline Target Cache (baseline) & 512-entry; \\
\hline SLB & 1k-entry store data array; \\
\hline
\end{tabular}

Listing 5. Identifying load/store instructions

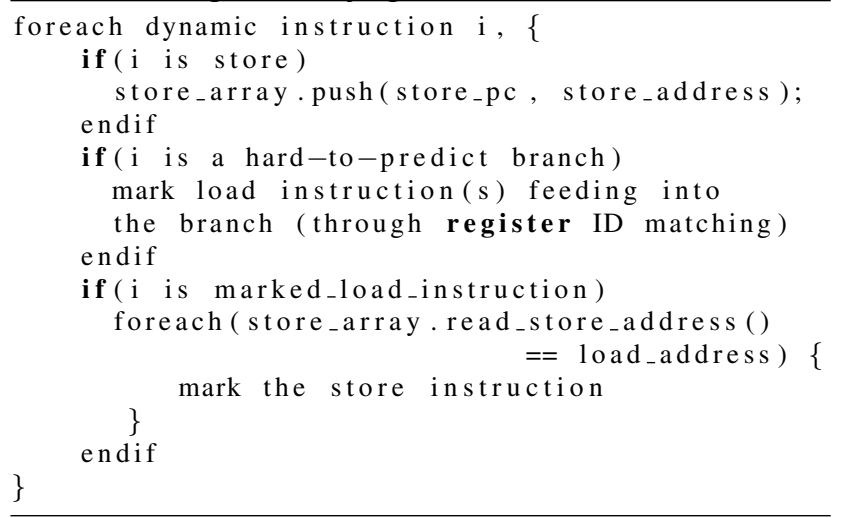

\subsection{Identifying Relevant Load/Store In- structions}

SLB predictor relies on compiler to identify program points where data structure associated with a data dependent branch is referenced and modified. For experiments in this paper, we have written a binary profiler for identifying load/store instructions associated with a data-dependent branch. Listing 5 shows pseudo code for our binary profiler.

\section{Results and Analysis}

\subsection{Store to Branch Delay}

SLB predictor uses store data for computing branch flags, therefore, store data should be available before branch instruction gets fetched. Table 4 shows cycle count between store data becoming available and branch flags computed using store data getting consumed. Following two program characteristics explain high cycle count between store-branch instruction pair. Firstly, data structure update and traversing typically happen in different program phases. Secondly, even when update and traversal are adjacent to each other, dynamic instructions created by update loop 


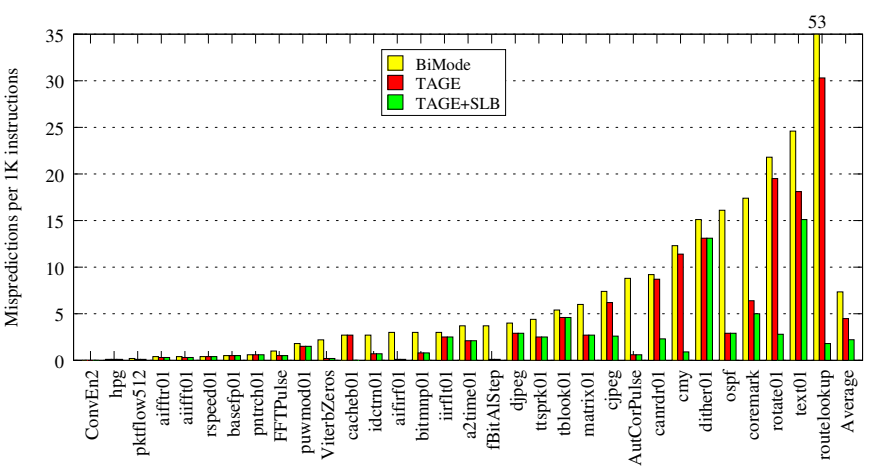

Figure 6. MPKI (EEMBC)

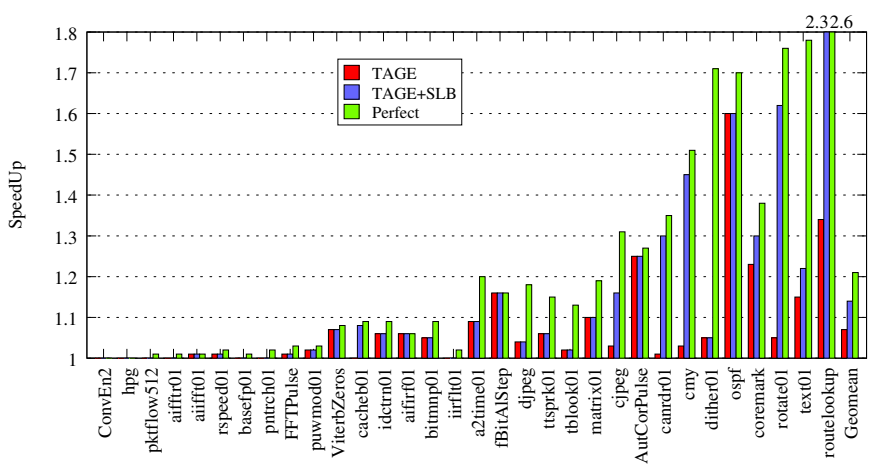

Figure 7. Speedup over baseline predictor. (EEMBC)

generates necessary cycles between the first store operation of update loop and the first load-branch operation of traversal loop.

\subsection{Branch MPKI}

Table 4 shows that only a handful of static branch instructions are predicted using SLB predictor. Yet, these hard-to-predict, data-dependent branches are top contributors to overall misprediction (see table 7 and 8 for top mispredicting branches in each benchmark). Figure 6 and 8 compares MPKI of baseline Bi-Mode predictor with 1) standalone TAGE predictor, and 2) combined TAGE+SLB predictor. As shown in these figures, MPKI remains high for several benchmarks even when using state-of-the-art TAGE predictor. On average, combined TAGE+SLB predictor reduces MPKI for EEMBC suite from 4.48 to 2.21, a reduction of $51 \%$. Similarly, for SPECint suite, MPKI is reduced from 9.56 to 7.50 , a reduction of $21 \%$.

Table 7 and 8 show up to 10 top mispredicting branches in benchmarks we have studied. In most cases, except coremark and text01, SLB predictor is able to accurately predict top mispredicting branches of Bi-Mode and TAGE predictor. Branches not handled by SLB predictor are marked as ' $x$ '. In coremark, top mispredicting branch is an indirect

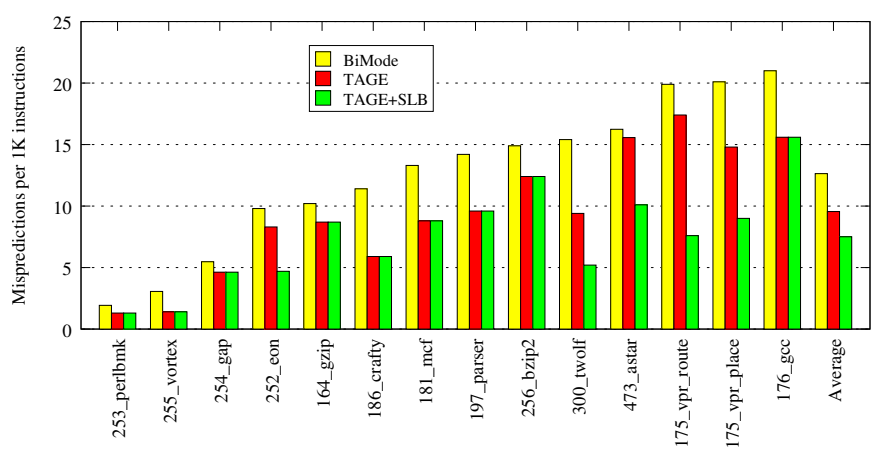

Figure 8. MPKI (SPECint)

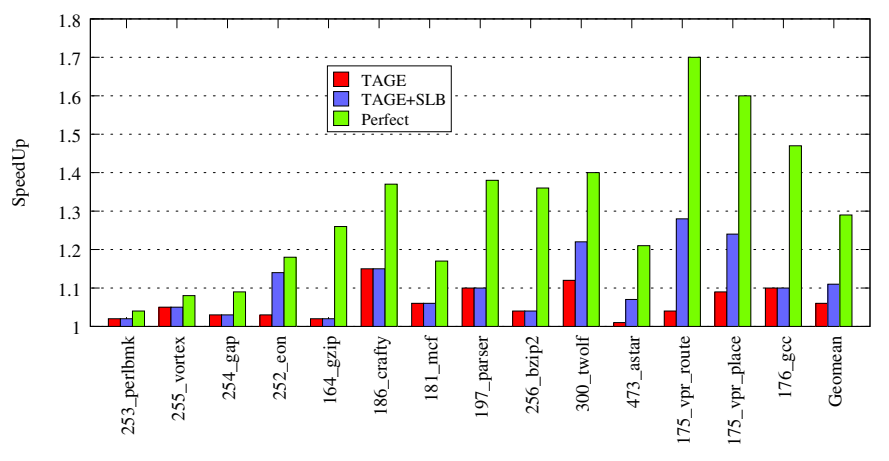

Figure 9. Speedup over baseline predictor. (SPECint)

branch resulting from a switch-case statement inside a for loop. In some case statements, control variable on which the indirect branch is dependent is also updated. This update is not available before next iteration of the for loop. As a result, SLB predictor reads stale value from store data array, therefore, mispredicts branch target address.

There are several benchmarks where combined TAGE+SLB predictor has same MPKI as that of the standalone TAGE predictor. This is because we have excluded these benchmarks from SLB analysis due to one of the following three reasons: 1) Benchmark MPKI is already low (e.g. EEMBC benchmarks on the left), 2) Mispredicting branches do not follow STLD-BR execution sequence (e.g. EEMBC/dither01, SPECint/164.gzip, SPECint/256.bzip2), and 3) Identifying ST-LD-BR sequence without compiler analysis is hard (e.g. SPECint/176.gcc, SPECint/197.parser).

\subsection{SLB Performance Impact}

Figure 7 and 9 compares performance improvement when using standalone TAGE predictor, combined TAGE+SLB predictor, and perfect predictor. For EEMBC suite, combined TAGE+SLB predictor doubled the speedup of standalone TAGE predictor over the baseline Bi-Mode predictor. A perfect predictor shows a potential of another 


\begin{tabular}{|c|c|c|c|c|c|c|c|c|c|c|c|c|c|}
\hline & $\begin{array}{c}\text { cacheb } \\
01\end{array}$ & cjpeg & $\begin{array}{c}\text { canrdr } \\
01\end{array}$ & cmy & $\begin{array}{l}\text { core- } \\
\text {-mark }\end{array}$ & $\begin{array}{c}\text { rotate } \\
01\end{array}$ & $\begin{array}{c}\text { text } \\
01\end{array}$ & $\begin{array}{c}\text { route } \\
\text { lookup }\end{array}$ & $\begin{array}{l}252 . \\
\text { eon }\end{array}$ & $\begin{array}{l}300 . \\
\text { twolf }\end{array}$ & $\begin{array}{c}473 . \\
\text { astar }\end{array}$ & $\begin{array}{c}\text { 175.vpr } \\
\text { route }\end{array}$ & $\begin{array}{c}\text { 175.vpr } \\
\text { place }\end{array}$ \\
\hline Num of SLB BR & 1 & 1 & 3 & 2 & 11 & 2 & 4 & 1 & 8 & 9 & 17 & 3 & 7 \\
\hline Num of SLB LD & 1 & 1 & 3 & 1 & 10 & 2 & 5 & 1 & 4 & 9 & 9 & 2 & 4 \\
\hline Num of SLB ST & 4 & 2 & 21 & 3 & 9 & 2 & 11 & 3 & 24 & 14 & 12 & 9 & 36 \\
\hline ST-BR Delay (cycles) & 1241 & 774 & 71 & 3626 & $16 \mathrm{~K}$ & 785 & 1004 & 53 & 799 & 556 & 256 & 534 & $1445 \mathrm{~K}$ \\
\hline
\end{tabular}

Table 4. Number of SLB-enabled branches, and associated load/store instructions

$7 \%$ performance improvement over the baseline. Similarly, for SPECint suite, combined TAGE+SLB predictor shows performance improvement of $11 \%$ over baseline Bi-Mode predictor. This is almost double the speedup of standalone TAGE predictor over the same baseline. Perfect predictor shows a potential for $29 \%$ performance improvement.

\subsection{SLB Area and Power Analysis}

Area and power overhead of SLB scheme was estimated using McPAT [22], a framework for modeling processor area and power. Parameters of a generic McPAT RISC core (Alpha21364) were adjusted to match simulated ARM core. TAGE and SLB predictor components were added for estimating their area and power overhead. Table 5 shows area and power overhead of SLB predictor for a $32 \mathrm{~nm}$ process technology node.

Table 5. SLB area and power overhead (at $32 \mathrm{~nm}$ )

\begin{tabular}{|c|c|c|c|c|c|}
\hline Metric & Core & Fetch Unit & TAGE & SLB & $\begin{array}{c}\text { SLB } \\
\text { overhead (\%) }\end{array}$ \\
\hline Area (mm2) & 13.99 & 1.64 & 0.10 & 0.07 & 0.5 \\
\hline Dy. Power (W) & 8.61 & 1.13 & 0.013 & 0.015 & 0.17 \\
\hline St. Power (W) & 6.66 & 0.74 & 0.023 & 0.028 & 0.42 \\
\hline
\end{tabular}

\subsection{SLB Timing Analysis}

Figure 10 shows critical timing paths in fetch unit. First two paths are through TAGE and SLB branch direction predictor, while the third path is through BTB for branch target address computation. Table 6 shows access time for various structures in fetch unit for a $32 \mathrm{~nm}$ technology process using CACTI [19].

Table 6. Access time for branch predictor structures

\begin{tabular}{|c|c|c|c|c|c|}
\hline & BTB & TAGE & \multicolumn{2}{|c|}{ SLB Tables } & \multirow{2}{*}{ MUX } \\
& & Component & Branch & T/NT & \\
\hline Access Time (ns) & 0.458 & 0.22 & 0.203 & 0.187 & 0.041 \\
\hline
\end{tabular}

- Access Time for TAGE Predictor (ns) $=$ TAGE component delay $+4 *($ MUX delay $)$ $=0.22+4 *(0.041)$

$=0.384$

- Access Time for SLB Predictor (ns)

$=$ Branch table delay $+\mathrm{T} / \mathrm{NT}$ table delay

$=0.203+0.187$

$=0.39$

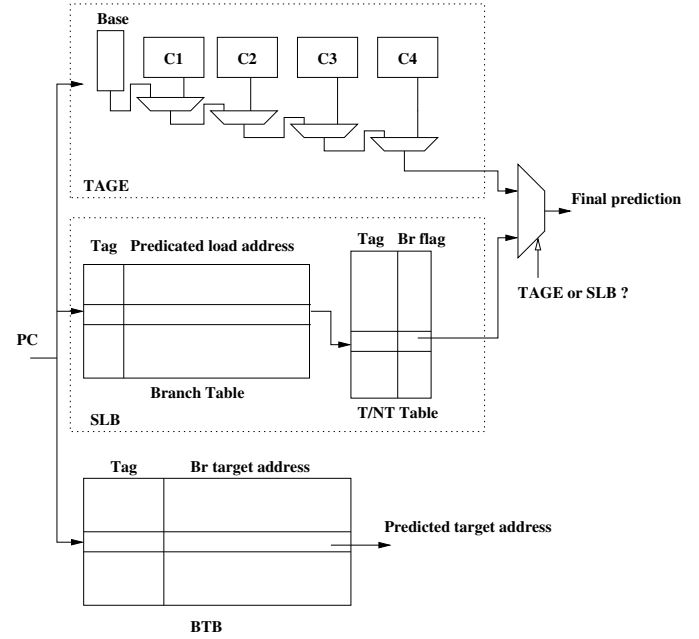

Figure 10. Comparing critical timing paths in fetch unit

- Access Time for BTB (ns)

$=$ BTB delay

$=0.458$

\subsubsection{Critical Timing Path in Fetch Unit}

Computing next instruction address after a branch instruction involves a) determining if branch is taken or not taken, i.e branch direction prediction, and b) if branch is indeed taken, computing branch target address. Therefore, critical path for fetch unit is the maximum delay between branch direction prediction and branch target address prediction, as shown below:

- Critical Timing Path in Fetch Unit (ns)

$=\operatorname{Max}\{$ Br. dir. pred. delay, Br. target pred. delay $\}$

$=\operatorname{Max}\{\operatorname{Max}\{$ TAGE, SLB $\}+$ mux delay, BTB delay $\}$

$=\operatorname{Max}\{\operatorname{Max}\{0.384,0.39\}+0.041,0.458\}$

$=\operatorname{Max}\{0.431,0.458\}$

$=\mathbf{0 . 4 5 8}$

\section{Related Work}

Branch prediction research can be categorized into three classes: static branch prediction, dynamic branch prediction, and compiler-assisted dynamic branch prediction.

Static Branch Predictors: In static branch prediction, branch direction is predicted before program is executed, 


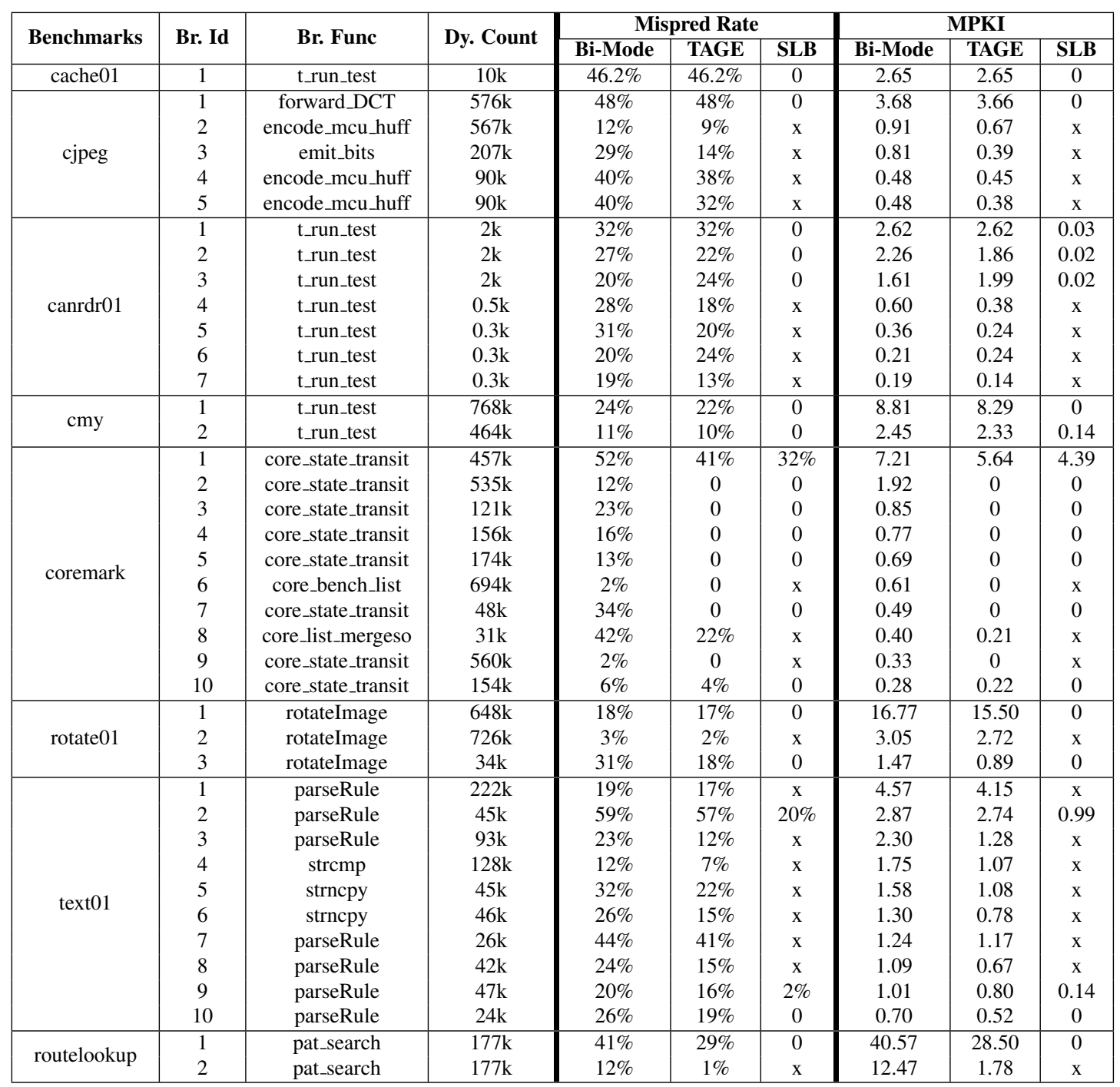

Table 7. Top mispredicting branches and their prediction accuracy for different predictors (EEMBC) 


\begin{tabular}{|c|c|c|c|c|c|c|c|c|c|}
\hline \multirow{2}{*}{ Benchmarks } & \multirow{2}{*}{ Br. Id } & \multirow{2}{*}{ Br. Func } & \multirow{2}{*}{ Dy. Count } & \multicolumn{3}{|c|}{ Mispred Rate } & \multicolumn{3}{|c|}{ MPKI } \\
\hline & & & & Bi-Mode & TAGE & SLB & Bi-Mode & TAGE & SLB \\
\hline \multirow{10}{*}{ 252.eon } & 1 & ggSpectrumSet & $20 \mathrm{~m}$ & $7 \%$ & $6 \%$ & 0 & 1.46 & 1.33 & 0 \\
\hline & 2 & ggGridIterator & $1789 \mathrm{k}$ & $37 \%$ & $35 \%$ & $\mathrm{x}$ & 0.68 & 0.65 & $\mathrm{x}$ \\
\hline & 3 & mrMaterialshad & $1232 k$ & $51 \%$ & $51 \%$ & $3 \%$ & 0.65 & 0.65 & 0.04 \\
\hline & 4 & ggGridIterator & $1537 \mathrm{k}$ & $38 \%$ & $36 \%$ & $\mathrm{x}$ & 0.60 & 0.58 & $\mathrm{X}$ \\
\hline & 5 & mrGridviewingH & $573 \mathrm{k}$ & $59 \%$ & $57 \%$ & $1 \%$ & 0.34 & 0.33 & 0 \\
\hline & 6 & mrGridviewingH & $1113 k$ & $28 \%$ & $2 \%$ & $\mathrm{x}$ & 0.32 & 0.02 & $\mathrm{x}$ \\
\hline & 7 & mrGridshadowHi & $634 \mathrm{k}$ & $48 \%$ & $46 \%$ & 0 & 0.31 & 0.30 & 0 \\
\hline & 8 & mrMaterialboun & $580 \mathrm{k}$ & $49 \%$ & $48 \%$ & $\mathrm{x}$ & 0.29 & 0.28 & $\mathrm{x}$ \\
\hline & 9 & mrSurfaceListv & $830 \mathrm{k}$ & $34 \%$ & $33 \%$ & $\mathrm{x}$ & 0.29 & 0.28 & $\mathrm{x}$ \\
\hline & 10 & mrGridviewingH & $950 \mathrm{k}$ & $26 \%$ & $20 \%$ & 0 & 0.25 & 0.20 & 0 \\
\hline \multirow{10}{*}{ 300.twolf } & 1 & new_dbox_a & $3144 k$ & $31 \%$ & $24 \%$ & 0 & 1.46 & 1.12 & 0 \\
\hline & 2 & new_dbox & $2473 k$ & $29 \%$ & $18 \%$ & 0 & 1.10 & 0.69 & 0.01 \\
\hline & 3 & new_dbox_a & $3175 \mathrm{k}$ & $23 \%$ & $15 \%$ & 0 & 1.08 & 0.74 & 0 \\
\hline & 4 & new_dbox_a & $3004 k$ & $20 \%$ & $13 \%$ & 0 & 0.91 & 0.58 & 0 \\
\hline & 5 & XPICK_INT & $780 \mathrm{k}$ & $62 \%$ & $1 \%$ & $\mathrm{x}$ & 0.73 & 0.01 & $\mathrm{x}$ \\
\hline & 6 & new_dbox_a & $1140 \mathrm{k}$ & $29 \%$ & $21 \%$ & 0 & 0.50 & 0.36 & 0 \\
\hline & 7 & new_dbox & $1084 \mathrm{k}$ & $26 \%$ & $7 \%$ & $\mathrm{x}$ & 0.42 & 0.12 & $\mathrm{X}$ \\
\hline & 8 & term_newpos & $928 \mathrm{k}$ & $25 \%$ & $18 \%$ & $4 \%$ & 0.35 & 0.25 & 0.06 \\
\hline & 9 & add_penal & $645 \mathrm{k}$ & $30 \%$ & $16 \%$ & $\mathrm{x}$ & 0.29 & 0.16 & $\mathrm{X}$ \\
\hline & 10 & term_newpos_a & $657 \mathrm{k}$ & $30 \%$ & $17 \%$ & 0 & 0.29 & 0.16 & 0 \\
\hline \multirow{10}{*}{ 473.astar } & 1 & makebound2 & $424 k$ & $34 \%$ & $34 \%$ & $3 \%$ & 1.20 & 1.21 & 0.11 \\
\hline & 2 & makebound 2 & $399 k$ & $28 \%$ & $24 \%$ & 0 & 0.93 & 0.81 & 0 \\
\hline & 3 & makebound 2 & $424 k$ & $25 \%$ & $22 \%$ & $12 \%$ & 0.90 & 0.79 & 0.43 \\
\hline & 4 & makebound2 & $393 k$ & $26 \%$ & $25 \%$ & $10 \%$ & 0.88 & 0.83 & 0.33 \\
\hline & 5 & makebound2 & $430 \mathrm{k}$ & $21 \%$ & $17 \%$ & $3 \%$ & 0.75 & 0.64 & 0.10 \\
\hline & 6 & makebound 2 & $400 \mathrm{k}$ & $22 \%$ & $20 \%$ & $6 \%$ & 0.74 & 0.67 & 0.21 \\
\hline & 7 & makebound 2 & $370 \mathrm{k}$ & $22 \%$ & $21 \%$ & $3 \%$ & 0.69 & 0.65 & 0.09 \\
\hline & 8 & makebound2 & $232 k$ & $35 \%$ & $33 \%$ & 0 & 0.68 & 0.64 & 0 \\
\hline & 9 & makebound2 & $390 \mathrm{k}$ & $18 \%$ & $16 \%$ & $13 \%$ & 0.59 & 0.53 & 0.44 \\
\hline & 10 & makebound 2 & $213 k$ & $32 \%$ & $30 \%$ & 0 & 0.57 & 0.54 & 0 \\
\hline \multirow{8}{*}{ 175.vpr_route } & 1 & route_net & $8365 \mathrm{k}$ & $32 \%$ & $36 \%$ & $4 \%$ & 5.19 & 5.84 & 0.75 \\
\hline & 2 & route_net & $11 \mathrm{~m}$ & $14 \%$ & $13 \%$ & 0 & 3.26 & 2.91 & 0.13 \\
\hline & 3 & node_to_heap & $4105 \mathrm{k}$ & $39 \%$ & $33 \%$ & $\mathrm{x}$ & 3.05 & 2.63 & $\mathrm{X}$ \\
\hline & 4 & route_net & $1247 \mathrm{k}$ & $80 \%$ & $3 \%$ & $\mathrm{x}$ & 1.91 & 0.08 & $\mathrm{x}$ \\
\hline & 5 & node_to_heap & $2982 k$ & $31 \%$ & $28 \%$ & $\mathrm{x}$ & 1.80 & 1.62 & $\mathrm{x}$ \\
\hline & 6 & route_net & $5084 \mathrm{k}$ & $16 \%$ & $17 \%$ & 0 & 1.64 & 1.66 & 0.07 \\
\hline & 7 & route_net & $939 \mathrm{k}$ & $40 \%$ & $41 \%$ & $\mathrm{x}$ & 0.73 & 0.75 & $\mathrm{X}$ \\
\hline & 8 & route_net & $8880 \mathrm{k}$ & $3 \%$ & $3 \%$ & $\mathrm{x}$ & 0.64 & 0.62 & $\mathrm{X}$ \\
\hline \multirow{10}{*}{ 175.vpr_place } & 1 & get_non_update & $4088 \mathrm{k}$ & $43 \%$ & $38 \%$ & $5 \%$ & 1.76 & 1.59 & 0.22 \\
\hline & 2 & try_swap & $3007 \mathrm{k}$ & $43 \%$ & $39 \%$ & $2 \%$ & 1.29 & 1.18 & 0.08 \\
\hline & 3 & get_non_update & $2321 \mathrm{k}$ & $44 \%$ & $40 \%$ & $10 \%$ & 1.02 & 0.94 & 0.23 \\
\hline & 4 & get_non_update & $2320 \mathrm{k}$ & $44 \%$ & $40 \%$ & $10 \%$ & 1.02 & 0.94 & 0.24 \\
\hline & 5 & try_swap & $2381 \mathrm{k}$ & $41 \%$ & $36 \%$ & 0 & 0.99 & 0.87 & 0 \\
\hline & 6 & try_swap & $3446 \mathrm{k}$ & $23 \%$ & $16 \%$ & 0 & 0.79 & 0.55 & 0 \\
\hline & 7 & update_bb & $1814 \mathrm{k}$ & $39 \%$ & $33 \%$ & $\mathrm{x}$ & 0.72 & 0.60 & $\mathrm{x}$ \\
\hline & 8 & try_swap & $3082 \mathrm{k}$ & $20 \%$ & $16 \%$ & $\mathrm{x}$ & 0.64 & 0.50 & $\mathrm{x}$ \\
\hline & 9 & get_bb_from_s & $2801 \mathrm{k}$ & $21 \%$ & $18 \%$ & $\mathrm{x}$ & 0.60 & 0.50 & $\mathrm{x}$ \\
\hline & 10 & get_bb_from_s & $2784 \mathrm{k}$ & $21 \%$ & $17 \%$ & $\mathrm{X}$ & 0.59 & 0.49 & $\mathrm{x}$ \\
\hline
\end{tabular}

Table 8. Top mispredicting branches and their prediction accuracy for different predictors (SPECint) 
and same prediction is used for all dynamic instances of that branch. Profiling is used in [13]. Simple heuristics such as predict all backward branches taken, and predict all forward branch not-taken were proposed in [33]. Machine learning was using in [5] to infer branch behavior of new program using existing programs.

Dynamic Branch Predictors: Dynamic branch prediction techniques propose hardware that attempts to learn branch behavior at run-time. While most dynamic schemes use branch taken/not-taken history information for training the branch predictor, few schemes have explored adding other information to the prediction process.

(i) Short History Predictors: James. E. Smith first presented bimodal branch prediction scheme [33]. Repeatedly taken branches will be predicted to be taken, and repeatedly not-taken branches will be predicted as not-taken. Two-level adaptive branch prediction scheme was presented in [37]. It is based on the observation that a branch can have multiple repetitive patterns. The two-level scheme differentiates among these patterns by keeping a record of direction taken by last $m$ instances of each branch, and using it to index into a table of k-bit counter. Combining branch predictor was proposed in [24] which combines and takes advantage of different predictors types.

(ii) Anti-aliasing Predictors: Several schemes proposed different indexing mechanism for reducing branch aliasing effect. gselect predictor concatenates branch history and branch address, and uses it to index into counter table [27]. gshare predictor uses exclusive OR of branch address with branch history to index into counter table [24]. gskewed predictor uses multiple counter tables indexed by different hash functions [26]. Bi-Mode [21] and YAGS [11] predictors partitioned counter table into two halves - taken and not-taken. It reduces negative interference by keeping branches biased towards 'taken' direction in the taken array, and those biased towards 'not-taken' direction in the not-taken array. Agree predictor [35] updates prediction counter based on whether or not branch bias matches branch outcome, irrespective of branch direction.

(iii) Long History Predictors: Recent research has shown that prediction accuracy can further be improved by utilizing longer branch history, and using different history length for predicting different branches. Using perceptrons instead of two-bit saturating counter was proposed in [16]. It is based on the observation that not all branches in history are important. O-GEHL [29], PPM-like [25] and TAGE [32] use multiple predictor tables, each indexed by an increasing length of branch history. In O-GEHL, final prediction is computed by summing predictions read from each predic- tor table. In PPM-like and TAGE final prediction is provided by the longest table that matches the tag.

(iv) Non-History Based Dynamic Branch Predictors: Available Register Value Information (ARVI) predictor [7] hashes register values with branch pc to index into prediction table. Branch prediction through Value prediction was proposed in [15]. Address-Branch Correlation (ABC) predictor [14] observed that values inside a data structure tend to be stable, therefore, branch outcome can be correlated simply with address of data structure instead of value inside data structure. We argue that if values inside data structure are stable, multiple iterations over data structure should generate same branch outcome every time, and should be predictable with a history-based predictor. Correlating load address with branch outcome is also used in [1], however, unlike [14], they update predictor in case there are stores to those addresses.

Compiler-Assisted Dynamic Branch Predictors: Compiler-assisted dynamic branch prediction combines strengths of static and dynamic approaches - the low overhead of compiler-time analysis with the effectiveness of dynamic prediction. Wish branch was proposed in [18]. It combines strengths of conditional branches and predication. Compiler generate code for predicated execution, but leaves conditional branches intact. At run-time, if branch turns out to be an easy-to-predict, branch prediction is used, else predicated code is executed. In [23], compiler defines a prediction function for each branch, and inserts instructions for computing prediction function.

\section{Conclusion and Future Work}

Data-dependent branches are single biggest source of remaining branch mispredictions. These branches are commonly associated with program data structures such as arrays, linked lists, trees etc., and follow store-load-branch execution sequence. A set of memory locations is written while building and updating the data structure. During data structure traversal, these locations are read, and used for evaluating branch condition. Branch outcome depends on data values stored in the data structure, which, typically do not have repeatable pattern. Therefore, in addition to history-based predictors, we need a different kind of predictor for data-dependent branches.

Taking advantage of store-load-branch execution sequence, we propose a compiler-assisted Store-Load-Branch (SLB) predictor. For every data-dependent branch, compiler identifies all store instructions that modify the data structure associated with the branch. These store instructions are dynamically tracked, and stored values are used for computing branch flags ahead of time. These branch flags 
are temporarily buffered in a hardware structure, and later used for making predictions. Section 5.2 shows that compared to standalone TAGE predictor, a hybrid TAGE+SLB predictor reduces branch MPKI by $21 \%$ for SPECint benchmark suite, and by $51 \%$ for EEMBC benchmark suite. Estimated power and area overhead of SLB predictor is $0.28 \%$ and $0.5 \%$ respectively (section 5.4 ), with no timing overhead (section 5.5).

Finally, we are working on a compiler implementation for identifying relevant load/store instructions using LLVM's Data Structure Analysis (DSA) [20]. In contrast to other alias analysis that operates on individual memory references, DSA operates at the level of entire instance of data structure, and provides context-sensitive $\mathrm{mod} / \mathrm{ref}$ analysis.

\section{References}

[1] M. Al-Otoom, E. Forbes, and E. Rotenberg. EXACT: Explicit Dynamic-Branch Prediction with Active Updates. In Proceedings of the 7th ACM international conference on Computing frontiers, CF' 10 , pages 165-176, New York, NY, USA, 2010. ACM.

[2] ARM. ARM Architecture Reference Manual. infocenter. arm. com.

[3] J. L. Baer and T. F. Chen. An Effective On-Chip Preloading Scheme to Reduce Data Access Penalty. In Proceedings of the 1991 ACM/IEEE conference on Supercomputing, Supercomputing '91, pages 176-186, New York, NY, USA, 1991. ACM.

[4] M. Bekerman, S. Jourdan, R. Ronen, G. Kirshenboim, L. Rappoport, A. Yoaz, and U. Weiser. Correlated Load-Address Predictors. In Proceedings of the 26th annual international symposium on Computer architecture, ISCA '99, pages 54-63, Washington, DC, USA, 1999. IEEE Computer Society.

[5] B. Calder, D. Grunwald, D. Lindsay, J. Martin, M. Mozer, and B. Zorn. Corpus-based Static Branch Prediction. In Proceedings of the ACM SIGPLAN 1995 conference on Programming language design and implementation, PLDI '95, pages 79-92, New York, NY, USA, 1995. ACM.

[6] P. Chang, E. Hao, and Y. N. Patt. Target Prediction for Indirect Jumps. In ISCA-24, pages 274-283, 1997.

[7] L. Chen, S. Dropsho, and D. H. Albonesi. Dynamic Data Dependence Tracking and its Application to Branch Prediction. In $H P C A$ 9 , page $65,2003$.

[8] T. F. Chen and J. L. Baer. Effective Hardware-based Data Prefetching for High-Performance Processors. IEEE Transactions on Computers, 44:609-623, 1995.

[9] K. Driesen and U. Hölzle. Accurate Indirect Branch Prediction. In ISCA-25, pages 167-178, 1998.

[10] K. Driesen and U. Hölzle. The Cascaded Predictor: Economical and Adaptive Branch Target Prediction. In MICRO-31, pages 249-258, 1998.

[11] A. N. Eden and T. Mudge. The YAGS Branch Prediction Scheme. In Proceedings of the 31 st annual ACM/IEEE international symposium on Microarchitecture, MICRO 31, pages 69-77, Los Alamitos, CA, USA, 1998. IEEE Computer Society Press.

[12] EEMBC. The Embedded Microprocessor Benchmark Consortium. http: //www. eembc.org.

[13] J. A. Fisher and S. M. Freudenberger. Predicting Conditional Branch Directions From Previous Runs of a Program. SIGPLAN Not., 27(9):85-95, Sept. 1992.

[14] H. Gao, Y. Ma, M. Dimitrov, and H. Zhou. Address-Branch Correlation: A Novel Locality for Long-Latency Hard-to-Predict Branches. In HPCA, pages 74-85. IEEE Computer Society, 2008.
[15] J. Gonzalez and A. Gonzalez. Control-flow Speculation through Value Prediction. IEEE Transactions on Computers, 50(12):13621376, 2001.

[16] D. A. Jiménez and C. Lin. Dynamic Branch Prediction with Perceptrons. In HPCA-7, page 197, 2001.

[17] J. Kalamatianos and D. R. Kaeli. Predicting Indirect Branches via Data Compression. In MICRO-31, pages 272-281, 1998.

[18] H. Kim, O. Mutlu, J. Stark, and Y. Patt. Wish Branches: Combining Conditional Branching and Predication for Adaptive Predicated Execution. In MICRO-38, pages 12 pp.-54, Nov. 2005.

[19] H. Labs. CACTI 5.3. http://quid.hpl.hp.com:9081/ cacti/.

[20] C. A. Lattner. Macroscopic Data Structure Analysis and Optimization. PhD thesis, Champaign, IL, USA, 2005. AAI3182303.

[21] C.-C. Lee, I.-C. K. Chen, and T. N. Mudge. The Bi-Mode Branch Predictor. In MICRO-30, pages 4-13, 1997.

[22] S. Li, J. H. Ahn, R. D. Strong, J. B. Brockman, D. M. Tullsen, and N. P. Jouppi. McPAT: An Integrated Power, Area, and Timing Modeling Framework for Multicore and Manycore Architectures. In Proceedings of the 42nd Annual IEEE/ACM International Symposium on Microarchitecture, MICRO 42, pages 469-480, New York, NY, USA, 2009. ACM.

[23] S. Mahlke and B. Natarajan. Compiler Synthesized Dynamic Branch Prediction. In Proceedings of the 29th annual ACM/IEEE international symposium on Microarchitecture, MICRO 29, pages 153-164, Washington, DC, USA, 1996. IEEE Computer Society.

[24] S. McFarling. Combining Branch Predictors. Technical Report TN36, Digital Western Research Laboratory, 1993.

[25] P. Michaud. A PPM-like, Tag-based Branch Predictor. In In Proceedings of the First Workshop on Championship Branch Prediction (in conjunction with MICRO-37, 2004.

[26] P. Michaud, A. Seznec, and R. Uhlig. Trading Conflict and Capacity Aliasing in Conditional Branch Predictors. In Proceedings of the 24th annual international symposium on Computer architecture, ISCA '97, pages 292-303, New York, NY, USA, 1997. ACM.

[27] S.-T. Pan, K. So, and J. T. Rahmeh. Improving the Accuracy of Dynamic Branch Prediction using Branch Correlation. In Proceedings of the fifth international conference on Architectural support for programming languages and operating systems, ASPLOS-V, pages 76-84, New York, NY, USA, 1992. ACM.

[28] RVCT. Realview Compilation Tools. http://www.keil.com/ arm/realview.asp.

[29] A. Seznec. Genesis of the O-GEHL Branch Predictor. http:// www.jilp.org/vol 7, 2005

[30] A. Seznec. The L-TAGE Branch Predictor. http://www. jilp. org/vol 9, 2007.

[31] A. Seznec and P. Michaud. Championship Branch Prediction. ftp: //ftp.irisa.fr/local/caps/TAGE.tar.gz.

[32] A. Seznec and P. Michaud. A Case for (Partially)-Tagged Geometric History Length Predictors. http://www. jilp.org/vol7, 2006.

[33] J. E. Smith. A Study of Branch Prediction Strategies. In Proceedings of the 8th annual symposium on Computer Architecture, ISCA '81, pages 135-148, Los Alamitos, CA, USA, 1981. IEEE Computer Society Press.

[34] SPEC. Standard Performance Evaluation Corporation. http:// www. spec.org.

[35] E. Sprangle, R. S. Chappell, M. Alsup, and Y. N. Patt. The Agree Predictor: A Mechanism for Reducing Negative Branch History Interference. In Proceedings of the 24th annual international symposium on Computer architecture, ISCA '97, pages 284-291, New York, NY, USA, 1997. ACM.

[36] T.-Y. Yeh and Y. N. Patt. Two-Level Adaptive Training Branch Prediction. In MICRO-24, pages 51-61, 1991.

[37] T.-Y. Yeh and Y. N. Patt. Alternative Implementations of Two-Level Adaptive Branch Prediction. In IN PROCEEDINGS OF THE 19TH ANNUAL INTERNATIONAL SYMPOSIUM ON COMPUTER ARCHITECTURE, pages 124-134, 1992. 